Research Paper

\title{
Successful Debridement of a Knee Joint Prosthesis Infected with Listeria Monocytogenes. Case Report and Review of Current Literature
}

\author{
Walter van der Weegen ${ }^{\circledR}{ }^{\circledR}$, Cees M. Verduin², Miriam Graumans ${ }^{3}$ and Henk J. Hoekstra ${ }^{1}$ \\ 1. Department of orthopedic surgery, St. Anna hospital, Bogardeind 2, 5664 EH, Geldrop, the Netherlands. \\ 2. Department of medical microbiology, Laboratory for Pathology and Medical Microbiology (Stichting PAMM), De Run 6250, 5504 DL, Veldhoven, the \\ Netherlands. \\ 3. Department of Infection control, St. Anna hospital, Bogardeind 2, 5664 EH, Geldrop, the Netherlands. \\ $\triangle$ Corresponding authors: Walter van der Weegen, St. Anna hospital, Bogardeind 2, 5664 EH, Geldrop, the Netherlands. Telephone number: ++31(0)40 2864280 \\ Fax number: ++31(0)40 2864281 E-mail: kog@st-anna.nl. \\ (c) Ivyspring International Publisher. This is an open access article distributed under the terms of the Creative Commons Attribution (CC BY-NC) license \\ (https://creativecommons.org/licenses/by-nc/4.0/). See http://ivyspring.com/terms for full terms and conditions.
}

Received: 2018.06.27; Accepted: 2018.09.27; Published: 2018.10.04

\begin{abstract}
According to the relevant literature, prosthetic joint infections caused by Listeria monocytogenes require two stage revision surgery or prosthesis removal for a successful outcome. We present the case of a patient who suffered such an infection after Total Knee Replacement surgery and was successfully treated with antibiotics, joint lavage, debridement and retention of the prosthesis.
\end{abstract}

Key words: Knee arthroplasty; Listeria monocytogenes; Debridement and Implant Retention

\section{Introduction}

Prosthetic joint infections caused by Listeria monocytogenes are rare and usually occur in immunocompromised patients. ${ }^{1}$ This can lead to grave consequences such as meningitis and sepsis. ${ }^{2}$ In case of prosthetic joint infection, the usual treatment is intensive antibiotics (AB) both intravenous (IV) and oral, combined with two-stage revision surgery or prosthesis removal.1, 3-8 Only 3 case reports have described joint lavage as a treatment in case of such an infection, each with positive outcomes but all with a rather short follow-up. ${ }^{9-11}$ We report the rare case of a Listeria monocytogenes infection of a prosthetic knee joint with a good outcome one year after treatment with antibiotics, surgical debridement, lavage and implant retention. We also present an overview of recent literature on this topic.

\section{Case presentation}

A 59 year old male was referred to our orthopedic clinic (March 2017) for severe osteoarthritis (OA) of the right knee and was scheduled for joint replacement surgery. BMI was $39 \mathrm{~kg} / \mathrm{m} 2$, he had a knee arthroscopy four years previous and the most recent intra-articular knee injections (3 hyaluronic acid injections) were 19 months ago. Earlier blood cultures in January 2017 were all negative. The history of the patient included anemia due to hemorrhoids which was surgically treated, hypertension since 2006, type 2 Diabetes Mellitus without insulin treatment since 2007, and spinal surgery for a herniated lumbar disc April 2017.

Staphylococcus aureus screening was positive and the patient started prophylactic treatment 5 days before surgery (daily nasal muporicin ointment and body and hair wash with disinfectant (chlorhexidine gluconate $4 \%$ )). Before surgery he also attended a counselling class on implications and possible complications of joint arthroplasty. At his hospital admission, the patient had no wounds, no apparent infection symptoms and no 
gastrointestinal tract symptoms. In May 2017 the patient received a total knee total prosthesis (Vanguard, Zimmer-Biomet, Warsaw USA) during an uneventful procedure. The prosthesis was cemented in place using Refobacin cement (Zimmer-Biomet, Warsaw USA) which contains both gentamicin and clindamycin. Other PJI prophylaxis included the administration of 2 grams Cefazolin before incision, skin disinfection using chlorhexidine $(0.5 \%$ in $70 \%$ alcohol) and extensive Sodium Chloride pulse lavage before the closing of the wound. The patient was mobilized on the day of surgery and discharged home the day after surgery without any fever and with minimal pain (Numeric Rating Score for pain on discharge: 2). Since minor wound leakage was present at discharge, the patient was provided with sufficient wound dressings and instructed on how to change the wound dressing daily.

Three weeks after discharge the patient was readmitted with a fever (39.2 Celsius) and severe knee pain. Gastrointestinal tract symptoms (including diarrhea in the previous weeks) were absent at readmission. Local knee temperature was increased with swelling present but no redness of the wound. C-reactive protein (CRP) was 167 milligram per litre $(\mathrm{mg} / \mathrm{L})$. Blood cultures upon readmission and 3 days later were all negative. During the Debridement, Antibiotics, Irrigation and Retention (DAIR) procedure one day later some pus was observed and a total of six cultures were taken from the implant interface and the synovial capsule. The polyethylene insert was exchanged, necrotic tissue removed as much as possible and a wound drain was left in place. Oral Flucloxacillin was given to the patient and one day later his pain had decreased, wound drainage was minimal $(140 \mathrm{ml})$ and his body temperature was 36.3 Celsius. All cultures were positive with a Gram-positive small rod-shaped bacterium, subsequently identified as Listeria monocytogenes. Subsequently, therapy was changed to amoxicillin 6 daily dose (dd) 2 grams IV. Flucloxacillin is not a preferred choice for Listeria monocytogenes, it is active, although much less than penicillin or amoxicillin. ${ }^{12}$ Furthermore, there are no recent data on susceptibility of Listeria monocytogenes to flucloxacillin or one of the other isoxazolyl penicillins. Serotype was determined by the Dutch National Reference Laboratory as 1/2a. A Peripheral Inserted Central Catheter (PICC) was inserted four days after the DAIR procedure allowing IV AB treatment for 14 days followed by oral Trimethoprim-sulfamethoxazole (Cotrimoxazol) $2 \mathrm{dd} 960 \mathrm{mg}$ for 10 weeks. In our institution amoxicillin and trimethoprim-sulfamethoxazol are acceptable choices for oral treatment of an infection with Listeria monocytogenes. It is up to the treating physician, in collaboration with the consultant microbiologist to make a choice.

One week after the DAIR procedure CRP was $49 \mathrm{mg} / \mathrm{L}$ and erythrocyte sedimentation rate (ESR) was $103 \mathrm{~mm} /$ first hour. To assess if the patient was immunocompromised he was tested for Human Immunodeficiency Virus (HIV) yet results were negative. Over the next days the patient was mobilised, his wound leakage further decreased and his clinical status improved considerably. He was discharged home two weeks after being readmitted, with CRP $19 \mathrm{mg} / \mathrm{L}$ and ESR 82 $\mathrm{mm}$ /first hour and with only some minor knee pain at night. One month later CRP was $2 \mathrm{mg} / \mathrm{L}$ and the patient was asymptomatic, even when walking or cycling. Although we initially intended to have $A B$ therapy for 6 months, the rapid clinical improvement with normalization of CRP and BSE within 4 weeks led us to reconsider and shorten the duration of $A B$ therapy. One year after the DAIR procedure the patient was asymptomatic, CRP and ESR were normal, and the prosthesis was functioning well.

Further detailed history taking revealed that the patient used to be a regular consumer of blue cheese made from unpasteurized milk, up to 3 times per week. This was continued in the weeks after the primary knee surgery. During this period the patient was also responsible for the daily changing of the wound dressing. Since more general listeria infection symptoms were absent (i.e. central nervous system infection), the combination of food consumption and wound care presents a possible infection route in this case. The patient has since reported that he no longer consumes any food associated with potential Listeria monocytogenes.

\section{Literature review}

Charlier et al. (2011) retrospectively summarized the clinical presentation and outcomes of Listeria monocytogenes infection surrounding an artificial joint, based upon 34 cases from the French National Reference Center database. ${ }^{1}$ They concluded that for the treatment of prosthetic infection with Listeria monocytogenes intensive $\mathrm{AB}$ 
treatment is required and that all patients who underwent implant removal or replacement were cured. In contrast, 5 of the 13 patients who did not undergo implant removal or replacement were reported as treatment failures. Bader et al. (2016) presented a case of late periprosthetic joint infection caused by Listeria monocytogenes which was treated with two-stage revision surgery and antibiotics, whereby the patient was doing well 5 months after reimplantation. They also reviewed the English literature on periprosthetic joint infections caused by Listeria monocytogenes and summarized 25 reported cases. In 15 of these cases the prosthesis was retained.13 All other published studies report on a single case. To our knowledge there are a few described cases of Listeria monocytogenes infected patients who were successfully treated with joint lavage, although in each case the maximum follow up duration was six months.9-11 Chougle et al. only used $A B$ in an infected elderly patient after hip joint replacement with a good outcome 18 months follow up. ${ }^{14}$ Since Morchon et al. reviewed the literature on prosthetic joint infection with Listeria monocytogenes up to 2007,7 we also summarized all literature published from a later date. We summarized all available literature not yet reviewed by either Morchon et al or Bader et al. See table 1.

\section{Discussion}

Infection with Listeria monocytogenes after joint replacement surgery is rare, but can lead to severe complications, including permanent joint removal. ${ }^{7}$ It is usually described as a late complication, manifesting itself months or years after the initial joint replacement surgery. ${ }^{10,} 13$ Usually patients with malignant disease or chronic conditions (i.e. diabetes, kidney or liver disease) are at risk, most often with a hematogenous route of infection. ${ }^{6}$ In our isolated case, the onset was within weeks, and taking into consideration that our patient was relatively young and that he was not immunocompromised besides having Diabetes Mellitus, performing a DAIR procedure became an alternative to a two-stage revision.

The listeria bacteria, which are small Gram-positive, non-spore-forming aerobic rods, is common in food products, typically in animal or vegetable products which are held at low temperature for an extended time and are eaten without being heated or cooked. In 2015, 47 cases of Listeria monocytogenes were registered in the Netherlands (type $1 / 2 \mathrm{a}: 45 \%$; type $1 / 2 \mathrm{~b} 13 \%$; type 4: $38 \%$ ). Several mechanisms (marked stimulation of the immune system with bone resorption as a result, survival in the macrophages (not responding to antibiotic therapy for which the bacterium is susceptible in vitro) and capability to form biofilm as well as settle in biofilms produced by other organisms) can make conservative management of Listeria infection difficult. ${ }^{5}$ To our knowledge, there is no research on the (clinical) efficacy of different antibiotics on biofilm formation produced by Listeria monocytogenes.

Although we cannot rule out the presence of a latent infection, our patient had no wounds or apparent infection symptoms at the time of hospital admission. Despite extensive prophylactic precautions and patient education, we cannot exclude that the route of wound infection is per continuitatem, though this pathogenesis is uncommon. In this case it demonstrates the importance of meticulous wound care after joint arthroplasty. We have shown that the treatment of a periprosthetic wound infected with Listeria monocytogenes using a DAIR procedure rather than a two-stage revision can be considered if the onset of infection symptoms is early and the patient is not immunocompromised. To strengthen the possibility of DAIR treatment, more reports on successful outcome of this treatment option are needed.

Table 1. Literature review

\begin{tabular}{|c|c|c|c|c|c|c|}
\hline Author (year) & N patients & Age & Immunocompromised & Joint & Treatment & Outcome \\
\hline Morchon $(2009)^{7}$ & 1 & 74 & Diabetes mellitus & Knee & 2-stage revision & Asymptomatic at follow up (duration unknown) \\
\hline Martinez-Pastor (2009) $)^{15}$ & 1 & 71 & No & Knee & 2-stage revision & Infection free at 24-month follow up \\
\hline Chrdle (2015) ${ }^{5}$ & 1 & 74 & Diabetes, COPD & Hip & 2-stage revision & Unknown \\
\hline Bader $(2016)^{13}$ & 1 & 57 & Diabetes & Knee & 2-stage revision & Asymptomatic at 5 months follow up \\
\hline
\end{tabular}




\section{Authors Contributions}

All authors contributed to study design and draft and/or revision of the manuscript.

\section{Competing Interests}

The authors have declared that no competing interest exists.

\section{References}

1. Charlier C, Leclercq A, Cazenave B, Desplaces N, Travier L, Cantinelli T, et al. Listeria monocytogenes-associated joint and bone infections: A study of 43 consecutive cases. Clin Infect Dis. 2012; 54: 240-8.

2. Allerberger F, Wagner M. Listeriosis: a resurgent foodborne infection. Clin Microbiol Infect. 2016; 16: 16-23.

3. Allerberger F, Kasten MJ, Cockerill FR, Krismer M, Dierich MP. Listeria monocytogenes infection in prosthetic joints. Int Orthop. 1992; 16: 237-9.

4. Breddam M, Hansen TB, Thornsen PB. Deep infection after primary hip arthroplasty: results after treatment of 10 patients. Eur J Orthop Surg Traumatol. 1996; 6: 101-3.

5. Chrdle A, Stárek M. Listeria infection of a prosthetic hip joint. Klin Mikrobiol Infekc Lek. 2011; 17: 62-6.

6. Mavrogenis AF, Savvidou OD, Vlasis K, Papagelopoulos PJ. Late Hip Arthroplasty Infection Caused by Listeria monocytogenes in a Non-Immunocompromised Patient. Surg Infect (Larchmt). 2011; 12: 137-40.

7. Morchón D, Tejedor M, Quereda C, Navas E, Meseguer M. Listeria monocytogenes prosthetic joint infection: Case report and review of the literature. Enferm Infecc Microbiol Clin. 2009; 27: 389-93.

8. Wollenhaupt J, Busche T, Zeidler H. Listeria monocytogenes Infection In a Prosthetic Knee Joint In Rheumatoid Arthritis During Methotrexate Therapy. J Clin Rheumatol. 1997; 3: 171-5.

9. Gómez Rodríguez N, Ibáñez Ruán J, González Pérez M. Prosthetic knee infection caused by Listeria monocytogenes in a woman with rheumatoid arthritis and Waldenström s macroglobulinemia. An Med Interna. 2006; 23 : 276-8.

10. Seo Y, Noh YS, Wie SH, Chang UI. Prosthetic knee joint infection due to Listeria monocytogenes bacteremia in a diabetic female. Korean J Int Med. 2016; 31: 616-9.

11. Weiler PJ, Hastings DE. Listeria monocytogenes--an unusual cause of late infection in a prosthetic hip joint. J Rheumatol. 1990; 17: 705-7.

12. Sutherland R, Croydon EA, Rolinson GN. Flucloxacillin, a new isoxazolyl penicillin, compared with oxacillin, cloxacillin, and dicloxacillin. Br Med J. 1970; 4: 455-60.

13. Bader G, Al-Tarawneh M, Myers J. Review of Prosthetic Joint Infection from Listeria monocytogenes. Surg Infect (Larchmt). 2016; 17: 739-44.

14. Chougle A, Narayanaswamy V. Delayed presentation of prosthetic joint infection due to Listeria monocytogenes. Int J Clin Pract. 2004; 58: 420-1.

15. Martínez Pastor JC, Casanova Mora L, Vilchez Cavazos F, Lozano Lizárraga L, Castillo García F, Soriano Viladomiu A. Listeria monocytogenes infection in a total knee arthroplasty. Acta Ortop Mex. 2009; 23: 302-5. 